\title{
Medical ultrasonics in urology
}

\author{
Yoshizo Itoh
}

(C) The Japan Society of Ultrasonics in Medicine 2012

The World Congress of Endourology and SWL (WCE) was held in Kyoto last autumn. This international conference focuses particularly on the field of endoscopic therapy as a subspecialty of urology. At the time of inception of the WCE, about 20 years ago, extracorporeal shockwave lithotripsy (ESWL) had just made its debut, and new endourological treatments using the nephroscope or ureteroscope were being developed and their use was spreading; at that time, therefore, many of the papers and presentations were centered on these topics. Later, as ESWL spread to medical facilities throughout the world, it came to be regarded as just another routine procedure, and the number of related papers and presentations decreased accordingly. In its place, laparoscopic surgery became the new focus of attention. Beginning with surgical techniques for the kidneys and adrenals, this surgical technique spread rapidly to other fields, and laparoscopic procedures are now the standard treatment for many types of surgical interventions. While laparoscopic surgery was initially performed using a number of different ports for insertion of the scope and forceps, laparo-endoscopic single-site surgery (LESS) has made its debut in the twenty-first century, and makes it possible to insert both the endoscope and forceps via the single port. We are also seeing the development of naturalorifice transluminal endoscopic surgery (NOTES), which enables surgeons to operate without creation of new wounds in the body, with the instruments inserted through natural orifices such as the anus, vagina, etc. Moreover, in the United States, laparoscopic surgery, which caught on very quickly for the treatment of prostate cancer, is already

Y. Itoh $(\bowtie)$

Department of Urology, Kyoto Second Red Cross Hospital,

355-5 Haruobi, Kamigyo, Kyoto 602-8026, Japan

e-mail:vanpra@aol.com being replaced by robotic-assisted surgical techniques, which are fast becoming mainstream. The turnover of surgical procedures has been dizzyingly rapid. Many of the presentations given at the WCE were related to the latest endoscopic procedures.

On the final day of the conference, lectures and critiques were presented by renowned urologists who are familiar to us as authors of textbooks. In one of the sessions, we heard the expressions "from scalpel to needle" and "from needle to nothing." In other words, we first saw a transition from conventional surgery performed with scalpels to puncture procedures using needles instead, later progressing to treatment techniques that do not even involve the use of needles, such as intensity-modulated radiation therapy (IMRT) for prostate cancer and high-intensity focused ultrasound (HIFU) for prostatic hyperplasia as well as prostate cancer. Of course, this being a conference on endoscopy, ultrasound for diagnosis/treatment was not addressed directly. Yet in the rush of changes in treatment methods, ultrasound has played a role at every turn, and since most puncture procedures are ultrasound-guided, and HIFU also uses ultrasound, I listened with great interest to any discussion on ultrasound from these standpoints.

If we turned our attention to medical ultrasonics for a moment, we would find that urologists make relatively few contributions to academic journals or conferences that address these techniques. Moreover, departments of urology have the smallest number of specialists certified by learned societies in this field. Of course, the fact that the number of urologists itself is small can be regarded as one reason for this, yet we must ask ourselves how we can encourage more urologists to gain membership of the Japan Society of Ultrasonics in Medicine. How can we have more urologists qualifying as ultrasound specialists? And how can we bring about a growth in the number of papers and 
presentations related to ultrasound in urology? Twenty years ago, we saw a far greater number of papers and presentations on such topics as transrectal ultrasonography, ultrasound-guided paracentesis, and Doppler ultrasound. These procedures have already become completely routine and incorporated into ordinary treatment programs, and no longer generate the buzz they once did. The years since have seen new technologies appear on the horizon, such as 3D ultrasonics, contrast media, elastography, and Global Positioning System (GPS); however, the number of related presentations in the field of urology still remains limited. The rekindling of interest in urological ultrasound-particularly among our young urologists-will require the emergence of new topics in ultrasound diagnosis and treatment that would have the kind of impact we once saw with the many previous presentations on transrectal ultrasound and puncture procedures, the lively outflow of presentations in the ESWL and endourology from the Japanese Society of Endourology, and the more recent swelling of interest in laparoscopes. It goes without saying that one of the big issues that we need to address now is the creation of compelling new topics that can spark such a response in the urologists of today. 